\title{
Knappe Ressourcen in der Katastrophe und erhöhte Anforderungen im intensivmedizinischen Alltag
}

\author{
Wolfgang Kröll
}

\section{Einleitung}

Das Auftreten eines außergewöhnlichen Ereignisses erfordert außergewöhnliche Mittel zu dessen Bewältigung. Die weltweite Verbreitung der Lungenerkrankung COVID-19 (Corona Virus Disease 2019) hat die Weltbevölkerung, insbesondere aber die politisch Verantwortlichen, in Angst und Schrecken versetzt und es wurden und es werden Maßnahmen getroffen, die möglicherweise weit über das Ziel hinausgeschossen haben ${ }^{1,2}$.

Unbestritten ist, dass sich zahlreiche Menschen im Rahmen dieser Pandemie (zu definieren als eine länder- und kontinentübergreifende Ausbreitung einer Infektionserkrankung) weltweit mit diesem Virus infiziert haben, dass zahlreiche Menschen weltweit verstorben sind - ob sie nun mit dem Virus oder am Virus verstorben konnte bislang flächendeckend nicht nachgewiesen werden -; unbestritten ist auch, dass manche infizierte Menschen eine intensivmedizinische Behandlung benötigt haben.

Nun ist das Niveau der medizinischen Behandlung weltweit unterschiedlich. Um dies bestätigt zu erhalten, braucht man nicht auf die Zahlen der sog. Drittländer zu blicken, man braucht den Blick nicht einmal über Europa, nicht einmal über Mitteleuropa hinaus richten. Vergleicht man nur Österreich mit Italien, hat man zwei benachbarte Staaten, die über wesentlich unterschiedliche Gesundheitseinrichtungen verfügen. Dies bedeutet, dass kranke Menschen bereits in diesen beiden Staaten auf unterschiedlichem Niveau behandelt wurden ${ }^{3}$. Die Krankheit, der Krankheitsverlauf und der Tod eines Menschen sind dann nicht mehr vergleichbar. Das aber hat niemand davon abgehalten hat, die Bevölkerung hierzulande mit der Annahme in Angst und Schrecken zu versetzen, dass, wenn die Ausbreitung in ähnlicher Weise voranschreiten würde wie in Italien, wir - die Bürgerinnen und Bürger eines Staates - zu befürchten hätten,

1 Vgl. Taupitz, Verteilung medizinischer Ressourcen in der Corona-Krise.

2 Vgl. Buyx, A./Lipp, V., Orientierungshilfen in der Corona-Krise.

3 Siehe dazu auch den Beitrag von Ploner in diesem Band. 
dass auch unser Gesundheitssystem über unzureichende intensivmedizinische Ressourcen verfüge und es daher zwangsläufig zu Triage-Situationen, d.h. einer Bewertung kranker Menschen im Hinblick auf die Zuteilung bzw. die Vorenthaltung von medizinischen Ressourcen, kommen müsste.

\section{Triage und ibre Auswirkungen}

\subsection{Triage allgemein}

Die Ankündigung in Zeiten einer Pandemie, in der viele Menschen betroffen sind, in der viele Menschen befürchten, infiziert zu werden, es werde bei Fortschreiten der Infektion zu einem Engpass an Spitalsbetten und insbesondere an Intensivbehandlungsbetten kommen und man werde daher die betroffenen, schwer erkrankten Menschen „triagieren“ müssen, d.h. man werde eine Auswahl treffen müssen, welchem Kranken man ein Intensivbett bzw. ein Beatmungsgerät zur Verfügung stellt, derartige Aussagen gleichen politischem Schwachsinn und bewirken nur eines: steigende Angst in der Bevölkerung. Denn zumindest für die potenziell betroffene Bevölkerung erscheint es so, dass man mit der „Triage“ nun ein neues Instrument zur Auswahl von kranken Menschen entwickelt, denen man eine medizinische Unterstützung zu teil werden lässt, während man andere Betroffene davon ausnimmt. Dieser Begriff und das damit verbundene Vokabular wurden einfach in den Medien platziert, ohne darauf hinzuweisen, dass es sich dabei um ein schon lange gebrauchtes Tool in der Patientenselektion im Kriegsfall und in Katastrophensituationen handelt, vor allem aber auch, ohne den potenziell Betroffenen mitzuteilen, nach welchen Kriterien man eine solche Evaluierung durchführen wird bzw. durchführen wird müssen ${ }^{4,5}$.

Triage ist nichts Neues. Die „Sichtung“ von Patienten im Sinn einer Auswahl, welche von ihnen man aufgrund beschränkter Ressourcen einer gezielten Behandlung zuführen kann, eine solche Vorselektion ist grundsätzlich seit Langem bekannt. Wahrscheinlich reicht sie bis an den Beginn der Menschheit zurück, es kann nur nicht bewiesen werden. Bewiesen werden hingegen kann, dass die Sichtung von Verwundeten und Schwerverletzten in der Militärgeschichte im Zusammenhang mit kriegerischen

4 Vgl. Lippelt et al., Vorbereitung auf medizinische Großschadensereignisse.

5 Vgl. Gretenkort, Begrenzte Ressourcen - die Allokationsproblematik beim Massenanfall von Berletzten. 
Auseinandersetzungen integraler Bestandteil der ärztlichen Versorgung von Soldaten war. Urkundlich erwähnt bereits im 16. Jahrhundert zieht sich diese Thematik herauf bis in das 20. Jahrhundert, wo das Thema der Sichtung von Verletzten im Zusammenhang mit der Entwicklung der Notfall- und Katastrophenmedizin erneut Bedeutung erlangte ${ }^{6}$.

Massenunfälle und Katastrophen stellten die Mitarbeiter von Rettungsdiensten schon immer vor eine enorme Herausforderung. Eine große Zahl von Verletzten unterschiedlichen Ausmaßes machte es notwendig, von einem individualmedizinischen Versorgungskonzept Abstand zu nehmen und Überlegungen anzustellen, wie man trotzdem eine maximale Zahl an betroffenen Menschen unter den gegebenen Bedingungen versorgen konnte.

Eine „Sichtung“ wird in diesem Zusammenhang definiert als die ärztliche Beurteilung und Entscheidung über die Priorität der medizinischen Versorgung von Patienten hinsichtlich Art und Umfang der Behandlung sowie über Zeitpunkt, Art und Ziel des Transportes. Damit wird aber auch ersichtlich, dass trotz eingeschränkter personeller, medikamentöser, materieller und weiterer organisatorischer Ressourcen es immer die medizinische Versorgung der verletzten und/oder erkrankten Menschen ist, die im Vordergrund und im Mittelpunkt einer medizinischen Behandlung, auch unter den Bedingungen des Massenanfalls von Verletzten und/oder einer Katastrophe, steht ${ }^{7}$.

Um bei einem Massenanfall von Verletzten und/oder erkrankten Personen aus dem Chaos der ersten Minuten und Stunden zu einem weitgehend geordneten Systemablauf zu kommen, bedarf es nun einmal auch der Kategorisierung dieser betroffenen Menschen. Primäres Ziel einer solchen Triage ist die Festlegung des Versorgungsauftrages und des Versorgungsumfangs - erst danach kann auch eine Festlegung der Transportpriorität erfolgen.

6 Vgl. Sefrin, Sichtung - zentrales Element zur Bewältigung eines Großschadens und einer Katastrophe.

7 Vgl. Sefrin, Sichtung - zentrales Element zur Bewältigung eines Großschadens und einer Katastrophe. 
Über die folgenden Versorgungs- resp. Sichtungskategorien herrscht Einstimmigkeit:

\begin{tabular}{|l|l|l|}
\hline I & $\begin{array}{l}\text { akute, lebensgefährdende } \\
\text { Bedrohung } \\
\text { schwer verletzt / erkrankt }\end{array}$ & $\begin{array}{l}\text { Sofortbehandlung } \\
\text { dringende Behandlung }\end{array}$ \\
\hline II & $\begin{array}{l}\text { spätere (ambulante) } \\
\text { Behandlung }\end{array}$ \\
\hline III & leicht verletzt / erkrankt & $\begin{array}{l}\text { betreuende / abwartende } \\
\text { Behandlung }\end{array}$ \\
\hline IV & ohne Überlebenschance & $\begin{array}{l}\text { Kennzeichnung / } \\
\text { Registrierung }\end{array}$ \\
\hline
\end{tabular}

\subsection{Triage als Schreckgespenst}

Das Erfordernis, mittels Triage bzw. mittels Sichtung knappe Ressourcen einer großen Menge an kranken und verletzten Menschen zuzuteilen, stellt für einen Krankenhausbetreiber zwar kein Schreckgespenst, dennoch aber eine ernst zu nehmende Herausforderung dar. Dass unerwartet und unangemeldet plötzlich eine Vielzahl an Patienten in der Notaufnahme einer Krankenanstalt eintrifft, die schließlich auch noch adäquat versorgt werden muss, gehört sicherlich nicht zum klinischen Alltag. Aber es gibt genügend Beispiele, dass solche Situationen existieren und sie bewältigt werden können.

Als ein Beispiel sei die Flugzeugkatastrophe von Ramstein genannt. Am 28. August 1988 kollidierten auf der von den Vereinigten Staaten in Ramstein bei Kaiserslautern (Rheinland-Pfalz) betriebenen Air Base während einer militärischen Flugschau drei Kunstflugmaschinen und stürzten daraufhin ab. Die Zahl der anwesenden Besucher wurde auf mehr als 300.000 geschätzt. Eines der Flugzeuge rutschte brennend ins Publikum, ein zweites traf einen in Notfallbereitschaft stehenden Hubschrauber. Dem Unglück fielen nach offiziellen Angaben 70 Menschen zum Opfer, 66 Flugtagbesucher, der Hubschrauberpilot sowie die drei Piloten der abgestürzten Flugzeuge. Zusätzlich gab es etwa 1000 Verletzte. Bei der überwiegenden Mehrzahl der Betroffenen kam es zu großflächigen Verbrennungen der Haut, die zum baldigen Tod oder zu Behinderungen bzw. Entstellungen, teilweise auf Lebenszeit, führten. Die Katastrophe von Ramstein ge- 
hört zu den folgenschwersten Katastrophen dieser Art weltweit und hatte weitreichende Konsequenzen für die Organisation des Notfallrettungswesens, die Opfer- und Helfernachsorge sowie die Durchführung von Flugschauen in Deutschland, Europa und den USA ${ }^{8}$.

Am 3. Juni 1998 ereignete sich auf der Bahnstrecke Hannover-Hamburg am Streckenkilometer 61 in der Gemeinde Eschede (Niedersachsen) ein Eisenbahnunfall infolge der Entgleisung des ICE 884 „Wilhelm Conrad Röntgen“. Dabei kamen 101 Menschen ums Leben, 88 wurden schwer und 106 Mitreisende leicht verletzt. Es handelte sich um den bislang schwersten Eisenbahnunfall in der Geschichte der Bundesrepublik Deutschland sowie um den schwersten Hochgeschwindigkeitszugunfall weltweit. Alle Schwerverletzten wurden innerhalb der nächsten Stunden in die umliegenden Krankenhäuser transportiert und konnten dort auch adäquat versorgt werden, obwohl, und dies soll als krasser Gegensatz zur Panikmache in der Corona-Krise herausgestellt werden, die Versorgung zu einem Zeitpunkt geschehen musste, an dem das operative Hauptprogramm des Tages abzuarbeiten war?.

Die Costa Concordia, ein so genanntes Post-Panamax-Kreuzfahrtschiff, war zum Zeitpunkt ihrer Indienststellung das größte Kreuzfahrtschiff Italiens. Es gehörte der italienischen Reederei Costa Crociere, die Teil des britisch-US-amerikanischen und weltgrößten Kreuzfahrtunternehmens, Carnival Corporation \& plc, ist. Am 13. Jänner 2012 kam es zu der bekannten Katastrophe vor der Insel Giglio mit ca. 1400 Einwohnern. Da dort nicht alle Passagiere untergebracht werden konnten, brachte man noch in der Nacht mit allen verfügbaren Fähren viele Gerettete auf das Festland nach Porto S. Stefano. Nach Abschluss der eigentlichen Bergungsaktion wurden im Lauf des 15. Januar noch drei Überlebende aus dem Schiffsrumpf gerettet, ungefähr 50 Personen wurden zu diesem Zeitpunkt vermisst. Rund dreißig Menschen wurden verletzt. 12 Personen starben noch während der Rettungsaktion oder wurden tot aus dem Meer geborgen. Für die weitere Suche nach Opfern wurde auch ein Tauchroboter eingesetzt. Im Verlauf der Suchaktion wurden bis Ende März 2012 weitere achtzehn Todesopfer im Wrack entdeckt. Damit belief sich die Zahl der gefundenen Leichen auf 30, die Mitte April alle identifiziert waren ${ }^{10}$.

$\mathrm{Zu}$ weiteren bisherigen Großschadensereignissen zählen u.a. der Absturz eines ungarischen Autobusses auf der Tauernstraße in Trieben

8 Vgl. Seelmann-Eggebert, Die Flammenhölle von Ramstein.

9 Vgl. Spiegel.de, ICE-Unglück von Enschede.

10 Vgl. Straub, Italiens Trauma nach der Havarie der Costa Concordia. 
(1991), das Bergwerksunglück in Lassing (1998), ein verunglückter Reisebus bei Windischgarsten, OÖ (1998), ein Murenabgang in Haus/E. (1999), ein Großbrand einer Diskothek in St. Agatha, OÖ (1999), ein Reisebusabsturz in der Nähe von Admont (2007), die Evakuierung des Kleinsölktals nach schweren Unwettern (2010), die Entgleisung eines Schnellzuges bei Trieben (2012), Unwetterkatastrophen im Paltental (Juni u. Juli 2012), sowie ein Schneechaos im Bezirk Liezen (Jänner 2019).

Alle diese Beispiele belegen, dass erstens Sichtung ein unumgängliches und wichtiges Instrument zur Versorgung einer Vielzahl von Verletzten und/oder Erkrankten ist und dass zweitens in keinem dieser Fälle die lokalen Krankenanstalten, trotz der großen Anzahl an mehr oder weniger gleichzeitig antransportierten Patientinnen und Patienten, mit der Grund-, aber auch nicht mit der intensivmedizinischen Versorgung überfordert waren.

\section{Der Umgang mit knappen Ressourcen}

Selbst wenn man davon ausgeht, dass Österreich und Deutschland zu den Ländern Europas gehören, die ihrer Bevölkerung beste Gesundheitsleistungen anbieten können, darf dennoch nicht übersehen werden, dass es auch hier nicht möglich ist, sich im Hinblick auf Gesundheitsleistungen einfach nach persönlichen Wünschen zu bedienen. Es sind vielmehr nicht nur die Ressourcen begrenzt, sondern auch die Gesellschaft hat in Form des Gesetzgeber bestimmte Grenzen in der Vergabe von Gesundheitsleistungen eingezogen, die jeden Rechtsträger eines Krankenhauses, sei es ein öffentlicher oder ein konfessioneller, aber auch jeden Arzt dazu verpflichten, Ressourcen nicht zu verschwenden, sondern gezielt den Vorgaben gemäß einzusetzen ${ }^{11}$.

\subsection{Ressource Intensivbett}

Die Corona-Pandemie hat wie ein Damoklesschwert das möglicherweise nicht verfügbare Intensivbehandlungsbett inklusive eines Beatmungsgerätes über der österreichischen, aber auch über der deutschen Bevölkerung schweben lassen. Rückblickend muss man sich folgerichtig der Frage stellen, ob es in Österreich und in Deutschland eine zu geringe Bettenkapazi-

11 Vgl. Allgemeines Sozialversicherungsgesetz (ASVG) idgF BGBl. I Nr. 31/2020. 
tät an Normal-, aber auch an Intensivbetten gab bzw. gibt. Letztlich geht es dabei um die Frage, ob der jeweilige Staat die medizinische Versorgung seiner Bürger gewährleisten kann.

Nun stellen Spitalsbetten im Allgemeinen und Intensivbehandlungsbetten im Besonderen in Österreich, aber auch in Deutschland, keinen limitierenden Faktor für eine dem Stand der medizinischen Wissenschaft entsprechende Behandlung von kranken Menschen dar. Dies ergab eine rezente, von der OECD im Zusammenhang mit der Corona-Pandemie veröffentlichte Studie ${ }^{12}$.

Österreich steht vielmehr im OECD-Schnitt vergleichsweise gut da, wenn es um Akut- und Intensivbetten in Krankenhäusern geht, wie eine weitere aktuelle OECD-Kurzstudie zur Corona-Pandemie zeigt ${ }^{13}$. Bei den Akutbetten liegt Österreich bei den verfügbaren Betten pro Einwohnerzahl auf Platz fünf der untersuchten Länder, bei den Intensivbetten gar auf Platz zwei hinter Deutschland.

Besonderes Augenmerk muss in Zusammenhang mit dem Coronavirus den Intensivbetten gelten, die zum Flaschenhals der medizinischen Versorgung werden könnten. Eine vorläufige Analyse der jüngsten öffentlich verfügbaren Daten deutet laut OECD darauf hin, dass die Kapazitätsunterschiede in zehn OECD-Ländern enorm sind: An der Spitze liegt Deutschland mit 33,3 Intensivbetten pro 100.000 Einwohnern, gleich dahinter liegt Österreich mit 28,9 Intensivbetten pro 100.000 Menschen, dahinter folgen die USA $(25,8)$ und Frankreich $(16,3)$.

Der OECD-Schnitt liegt bei 15,9. Schlusslichter sind Italien $(8,6)$, Dänemark $(7,8)$ und Irland $(5,0)$. Anzumerken ist, dass für diese Statistik unterschiedliche Jahreszahlen herangezogen werden mussten. Während der Stand in Italien aktuell ist, musste für Dänemark die verfügbare Zahl aus dem Jahr 2014 herangezogen werden, die österreichischen Kapazitäten gibt die OECD mit Stand 2018 an ${ }^{14}$.

Diese Zahlen beweisen doch eindeutig, dass weder in Österreich, noch in Deutschland, Intensivbehandlungsbetten Mangelware darstellen. Zu jedem Zeitpunkt der Corona-Pandemie standen in Österreich und in Deutschland der hiesigen Bevölkerung ausreichend Normal- und Intensivbetten zur Verfügung, die darüber hinaus nur zu einem geringen Prozentsatz genutzt wurden. In Österreich waren während der Krise maximal $25 \%$

12 Vgl. OECD, Hospital beds.

13 Vgl. OECD, Beyond Containment.

14 Vgl. Gesundheit Österreich, Das österreichische Gesundheitswesen im internationalen Vergleich. 
der zur Verfügung stehenden Intensivbehandlungsbetten belegt. Deutschland konnte französische Patienten aufnehmen, die in diesem Land wegen eines Mangels an Intensivbetten nicht adäquat behandelt werden konnten und auch Österreich hat italienischen Staatsbürgern Intensivbetten für eine Akutbehandlung zur Verfügung gestellt.

Nun ist auf den Unterschied zwischen dem Massenanfall an Verletzten und Kranken bei einer Katastrophe und im Rahmen einer Pandemie wie der Corona-Krise hinzuweisen. In Katastrophenfällen ereignet sich der Anfall an verletzten und erkrankten Menschen akut und ist nicht vorhersehbar. Auch wenn sich solche Vorfälle in Simulationen durchspielen lassen, gibt es hier immer eine Differenz zum tatsächlichen Ernstfall und seiner Realität, und sei es nur eine hinzukommende, widrige Wetterlage.

Dagegen war die Corona-Krise weitgehend planbar. Es war lange vorher erkennbar, dass diese Virusinfektion auch in Österreich und in Deutschland auftreten wird und es war daher auch möglich, den Routinebetrieb von Krankenhäusern entsprechend zu reduzieren und damit eine ausreichende Zahl an Normal- und Intensivbetten zur Verfügung zu stellen, Betten, die rückblickend jedoch zu keiner Zeit benötigt wurden ${ }^{15}$.

All dies zeigt nochmals, dass es unsinnig war, das Thema Triage in unseren Ländern überhaupt anzusprechen. Es war durch die Entwicklung der Infektion nämlich frühzeitig erkennbar, worauf auch zahlreiche Wissenschaftler hingewiesen hatten, dass in Österreich und in Deutschland keine höhere Zahl an Intensivbetten benötigt würde.

\subsection{Knappe Ressource Personal}

Mitarbeiterinnen und Mitarbeiter in Gesundheitsberufen sind grundsätzlich eine knappe Ressource. Die Ursachen dafür sind multifaktoriell und können in diesem Zusammenhang nicht ausführlich dargestellt werden. Das Problem wurde von Verfasser bereits in einem anderen Zusammenhang ausführlich diskutiert ${ }^{16}$.

Auch wenn die Dichte an Ärzten und Pflegepersonen, die der österreichischen Bevölkerung zur Verfügung steht, im Vergleich zum europäischen Durchschnitt sehr beruhigend wirkt, ist dennoch festzuhalten, dass im Hinblick auf den klinischen Alltag hier keineswegs von einem Überan-

15 Siehe dazu auch das Interview von Kröll mit Tax, Falzberger und Köle in diesem Band.

16 Vgl. Kröll, Allokationsentscheidungen am täglichen Arbeitsplatz. 
gebot gesprochen werden kann. Sowohl im intra- wie im extramuralen Bereich kann es vielmehr in gewissen Bereichen sehr wohl zu einer Knappheit kommen.

Ganz anders jedoch im Rahmen der derzeit abgelaufenen Pandemie. Wie sich aus den Aussagen des Direktoriums einer Universitätsklinik ergibt $^{17}$, bestand zu keinem Zeitpunkt der klinischen und medizinischen Krisenbewältigung ein Mangel an Ärzten und Pflegepersonen. Ganz im Gegenteil - weil während der Pandemie elektive Operationen und auch andere geplante und planbare medizinische Behandlungen kaum durchgeführt wurden, stand immer ausreichend Personal für die Behandlung und Betreuung von infizierten Menschen zur Verfügung. Zahlreiche Mitarbeiter mussten sogar nicht täglich an ihren Arbeitsplatz kommen, sondern standen als Reserve für den Fall zur Verfügung, dass in der Behandlung und Betreuung eingesetztes Personal akut erkrankt wäre. Obwohl dies auch geschah, konnte von der Führung des Krankenhauses doch gewährleistet werden, dass alle stationären, aber auch alle ambulant versorgten, Patienten dem Stand der medizinischen Wissenschaft entsprechend behandelt und betreut werden konnten.

\subsection{Knappe Ressource Arzneimittel und Medizinprodukt}

Der Massenanfall von infizierten und erkrankten Menschen, noch dazu in einer derart großen Anzahl, wie es bei der Erkrankung COVID-19 der Fall war, mag verständlicherweise auch Befürchtungen schüren, es könnten die erforderlichen Medikamente und Medizinprodukte, die einerseits für die Behandlung der Infektion, andererseits für die Behandlung und Betreuung chronisch kranker Menschen benötigt werden, zu einem knappen Mittel werden. Eine derartige Befürchtung war und ist nicht vollkommen unbegründet.

Wie aus den von Kröll und Platzer geführten Interviews hervorgeht, kam es zumindest am Beginn der Ausbreitung der Infektion, angesichts der von der Bundesregierung angeordneten Präventivmaßnahmen, zu Engpässen. Mundmasken für die Bevölkerung und entsprechende Schutzausrüstung für das medizinische Personal waren sowohl in den Krankenhäusern als auch in den Ordinationen niedergelassener Allgemeinmediziner und Fachärzte Mangelware. Erst allmählich ist es gelungen, derartige Schutzausrüstungen in Österreich selbst zu fertigen, insbesondere für das

17 Siehe dazu das Interview von Kröll mit Tax, Falzberger und Köle in diesem Band. 
medizinische Personal. Denn bereits der Antransport aus dem nahegelegenen Ausland wurde zum Problem. Der Lieferstopp bereits bestellter Medizinprodukte aus der Bundesrepublik Deutschland wurde bekanntlich damit begründet, dass man die Schutzausrüstungen für das eigene medizinische Personal benötige, was zeigt, dass in der Krise sich jeder selbst der Nächste ist.

Dass Ähnliches sich bei der Lieferung bzw. dem Verfügung-Stellen von Medikamenten ereignet hätte, ist nicht bekannt. Dennoch ist es durchaus denkbar, dass auch Medikamente Mangelware werden. Der entscheidende Grund dafür liegt darin, dass - aus ökonomischen Überlegungen - die Produktion von Medikamenten bzw. die Herstellung der Grundstoffe, die zur Produktion benötigt werden, vielfach in asiatische Staaten ausgelagert wurde. Im Rahmen einer kriegerischen Auseinandersetzung bzw. einer pandemischen Infektion wäre es dann durchaus denkbar, dass auch dringend benötigte Medikamente Mangelware werden könnten. Vergleichbares gilt auch für die erforderliche Durchführung klinischer Studien, die zur Einführung von Medikamenten notwendig sind und die ebenfalls vielfach nicht mehr in Europa, sondern z.B. in Asien durchgeführt werden. Es geht dabei nicht um Zweifel an der Korrektheit des Studiendesigns, sondern darum, dass Zulassungen dieser Medikamente in Europa entweder überhaupt nicht oder nur sehr zeitverzögert möglich sind, wenn die entsprechenden Studien nicht vorliegen ${ }^{18}$.

Man sollte daher die Corona-Pandemie zum Anlass nehmen, aus gesellschaftspolitischen, aber auch aus wirtschaftlichen Gründen zu überlegen, die Produktion von Grundstoffen, die man für die Herstellung von Medikamenten benötigt, aber auch die Produktion von Medizinprodukten, die für die Aufrechterhaltung der Gesundheit im Sinne von Prävention und Behandlung erforderlich sind, wiederum stärker nach Europa zu verlegen. Europa war einmal mit an der Spitze, was die innovative Produktion von Medikamenten und Medizinprodukten betrifft. Es gibt keinen Grund außer Gewinnstreben -, dass dies in Zukunft nicht wieder möglich wäre.

\section{Knappe Ressourcen im klinischen Alltag}

Die Diskussion um Sichtung und Triage verliert ihre gegenwärtige Brisanz im Zusammenhang mit der Covid-19 Pandemie, wenn man bedenkt, dass wertende Entscheidungen in der Behandlung kranker Menschen von Ärz-

18 Vgl. Laslop, Arzneimittelmarkt und Gerechtigkeit. 
tinnen und Ärzten unterschiedlichster Fachrichtungen täglich durchgeführt werden müssen ${ }^{19,20}$.

\subsection{Knappe Ressource Intensivbett}

Ungeachtet der Ausführungen in Abschnitt 3.1 können Intensivbehandlungsbetten in den Krankenanstalten Österreichs im klinischen Alltag immer wieder zu einer knappen Ressource werden. Der Grund liegt darin, dass Menschen mit unterschiedlichen Erkrankungen, Verletzungen und Vergiftungen sehr häufig, zumindest für den Zeitraum der akuten Erkrankungsphase, eine Behandlung und Betreuung auf einer Intensivstation benötigen. Dazu kommen Patienten, die sich ausgedehnten operativen Eingriffen zu unterziehen haben und hier nochmals die Gruppe älterer und damit in vielen Fällen multimorbider Menschen. Für Letztere gehen medizinische Behandlungen resp. operative Eingriffe allgemein mit einer Erhöhung des perioperativen Risikos einher und sie benötigen daher, wenn auch nur für einen überschaubaren Zeitraum, ein Intensivbehandlungsbett und möglicherweise auch eine Beatmungsmaschine.

Damit können auch scheinbar in genügender Anzahl verfügbare Intensivbehandlungsbetten situationsbedingt plötzlich zu einer knappen Ressource werden. Der behandelnde und verantwortliche Intensivmediziner hat dann an Hand bestimmter Kriterien zu entscheiden, welchem Patienten das verfügbare Intensivbehandlungsbett zugesprochen werden kann, was bedeutet, dass entweder ein zweiter Patient nicht auf eine Intensivstation verlegt werden kann, oder ein anderer die Intensivstation mangels ausreichender Bettenanzahl verlassen muss. Das dadurch möglicherweise die Heilungschancen eines Patienten gemindert werden, liegt auf der Hand und wird auch im klinischen Alltag in Kauf genommen ${ }^{21}$.

Eine Alternative wäre z.B., einem Patienten einen elektiven Eingriff, den er dringend benötigt, vorzuenthalten. Wird er aber, obwohl er den Eingriff dringend benötigt, von der Liste der zu operierenden Patienten gestrichen und für längere Zeit auf eine Warteliste gesetzt, hat dies zur Folge, dass er möglicherweise längere Zeit starke Schmerzen erleiden muss oder sogar auf der Warteliste verstirbt. Auch diese Entscheidung muss vom

19 Vgl. Christ et al., Bedeutung der Triage in der klinischen Notfallmedizin.

20 Vgl. Groening, Der alte Mensch im Notfallzentrum.

21 Vgl. Prien, Bestimmung des Therapieausmaßes. 
behandelnden und verantwortlichen Arzt getroffen und die Folgen müssen von ihm in Kauf genommen werden ${ }^{22}$.

Man steht hier vor Situationen außerhalb einer Katastrophe und außerhalb einer Pandemie, in denen Entscheidungen erforderlich sind, die möglicherweise zuungunsten eines Patienten, im schlimmsten Fall zuungunsten beider betroffenen Patienten ausgehen. Denn es ist ja keineswegs sicher, dass der Krankheitsverlauf für jenen Patienten, der weiter im Intensivbehandlungsbett betreut wird, sich günstig gestaltet.

\subsection{Knappe Ressource Personal}

Im klinischen Alltag der durchschnittlichen Kliniken und Krankenhäuser gibt es derzeit kaum Personalreserven. Vielmehr ist die Personalausstattung in den ärztlichen und nicht-ärztlichen Gesundheitsberufen knapp. Das hat zur Folge, dass Ausfälle an Personal, aus welchen Gründen auch immer, vom vorhandenen Personal kompensiert werden müssen. Dass dies weder zum Vorteil für die medizinische und pflegerische Behandlung und Betreuung der Patienten ist, noch zur Zufriedenheit der Mitarbeiter beiträgt, liegt auf der Hand. Kurzfristig sind derartige Kompensationen natürlich möglich und werden von den Mitarbeitern auch toleriert und mitgetragen, längerfristig jedoch führt es zur Unzufriedenheit, Demotivation und Burn-out der Betroffenen.

\subsection{Knappe Ressource Medikament}

Auch bei der Verabreichung von teuren Medikamenten können verantwortliche Ärzte dazu gezwungen sein, zu entscheiden, welchem Patienten - bzw. ob einem bestimmten Patienten überhaupt - diese Medikamente appliziert werden sollen. Diese Verantwortung überträgt der Gesetzgeber an den behandelnden Arzt in Form der Prämisse, dass eine Krankenbehandlung ausreichend und zweckmäßig sein muss und dass sie das Maß des Notwendigen nicht überschreiten darf ( $\mathbb{1 3 3}$ ASVG idgF). Diese Vorgabe ist keineswegs nur abstrakte Theorie, vielmehr wurde erst vor Kurzem einem minderjährigen Patienten, der an einer wahrscheinlich unheilbaren Muskelerkrankung litt, ein Medikament verweigert, welches pro Anwendung

22 Vgl. Heppner et al., Intensivmedizin im Alter. 
etwa $€$ 70.000.- kostet. ${ }^{23}$ Die Begründung lautete, dass die wiederholte Anwendung dieser Substanz weder eine Heilung noch eine Verbesserung der derzeitigen Situation ermöglichen werde. Mit anderen Worten kam es hier zu einer Triage im klinischen Alltag zu Lasten eines schwer kranken Patienten, ausgelöst durch eine entsprechende Rechtsnorm.

\section{Zusammenfassung}

Das Corona-Virus hat in den letzten Monaten sowohl im gesellschaftlichen Leben der Menschen, als auch das Gesundheitssystem vieler europäischer Länder betreffend, Einiges verändert. Ein laues Lüftchen einer grippalen Infektion ist zum Hurrikan einer Pandemie angewachsen. Zahlreiche Menschen sind mit dem Virus infiziert oder auch an der Viruserkrankung verstorben. Wie weit es dabei zu einer Übersterblichkeit im statistischen Sinn gekommen ist, werden später durchzuführende Berechnungen ergeben, für Österreich trifft dies zumindest laut Auswertung des statistischen Zentralamts bislang nicht zu.

Die eher martialische Kommunikation mancher Politiker hat zumindest Teile der Bevölkerung erheblich verängstigt. Welcher Einwohner eines Staates möchte hören, dass für ihn möglicherweise kein Intensivbehandlungsbett und keine Beatmungsmaschine zur Verfügung stehen, sollte er sie benötigen; und dies, obwohl sich Österreich und Deutschland rühmen, über die besten Gesundheitssysteme Europas zu verfügen.

Der vorliegende Beitrag wollte an Hand von Beispielen aufzeigen, dass die Verfahren von Triage, bzw. Sichtung und Vorselektion von Patienten bei einem Massenanfall nicht Ungewöhnliches und nichts Neues darstellen. Es handelt sich vielmehr um Instrumente, die es ermöglichen, in solchen Situationen die größte Anzahl an Menschenleben zu retten. Triage gehört in Wirklichkeit zum Arsenal jedes Notarztes, aber auch jedes klinisch tätigen Arztes; sowohl in der Notfallmedizin als auch im klinischen Alltag sind derartige Entscheidungen an der Tagesordnung. Und es bedarf dazu keineswegs des Anfalls einer großen Menge an erkrankten und/oder verletzten Menschen. In manchen Fällen, wenn z. B. nur ein Transportmittel zur Verfügung steht, können bereits zwei verletzte, verwundete oder erkrankte Menschen eine Entscheidung darüber erzwingen, welchen von ihnen man in die nächstgelegene Versorgungseinheit transportiert und welchem man damit eine größere Überlebenschance einräumt.

23 Vgl. Steiermark.orf.at, Streit um Therapiekosten für schwerkranken Buben. 


\section{Literatur}

Allgemeines Sozialversicherungsgesetz (ASVG) idgF BGBl. I Nr. 31/2020 (NR: GP XXVII IA 483/A AB 120 S. 27. BR: 10293 AB 10300 S. 906.), in: www.ris.bka.gv.a $\mathrm{t}$ [28. 05. 2020].

Buyx, A./Lipp, V.: Orientierungshilfen in der Corona-Krise - Die Ad-hoc-Empfehlung des Deutschen Ethikrates und die Klinsich-ethischen Empfehlungen von sieben Fachgesellschaften aus der Medizin, in MedR 38 (2020) $433 \mathrm{f}$.

Christ, M./Bingisser, R./Nickel, H.C.: Bedeutung der Triage in der klinischen Notfallmedizin, in Dtsch Med Wochenschr 141 (2016) 329-335.

Gesundheit Österreich GmbH (Hg.): Das österreichische Gesundheitswesen im internationalen Vergleich, in: www.sozialministerium.at/Themen/Gesundheit/Ges undheitssystem [28.05.2020].

Gretenkort, P.: Begrenzte Ressourcen - die Allokationsproblematik beim Massenanfall von Berletzten, in Notarzt 28 (2012) 211-214.

Groening, M.: Der alte Mensch im Notfallzentrum. Besondere Anfordernisse einer häufig multimorbiden Patientengruppe, in: Klinikarzt 47 (2018) 592-594.

Heppner, H.J./Sieber, C./Singker, K.: Intensivmedizin im Alter, in: Dtsch Med Wochenschr 138 (2013) 176-179.

Kröll, W.: Allokationsentscheidungen am täglichen Arbeitsplatz, in: Platzer, J./ Kröll, W. (Hg.): Gerechte Medizin? Analysen und Impulse aus Theorie und Praxis (= Bioethik in Wissenschaft und Gesellschaft 4), Baden-Baden: Nomos 2018, 111-130.

Laslop, A.: Arzneimittelmarkt und Gerechtigkeit, in: Platzer, J./Kröll, W. (Hg.): Gerechte Medizin? Analysen und Impulse aus Theorie und Praxis (= Bioethik in Wissenschaft und Gesellschaft 4), Baden-Baden: Nomos 2018, 147-164.

Lippelt, B./Günther, A./Boekhoff, J./Richter, C./Giezelt, M./Kohlmann, M.: Vorbereitung auf medizinische Großschadensereignisse. Abschätzung der zu erwartenden Patientenverteilung, in Notarzt 28 (2012) 203-210.

OECD: Beyond Containment: Health systems responses to COVID-19 in the OECD, in: http://www.oecd.org/coronavirus/policy-responses/beyond-containm ent-health-systems-responses-to-covid-19-in-the-oecd-6ab740c0/ [23.06.2020].

OECD: Hospital beds (indicator), doi: 10.1787/0191328e-en, in: https://data.oecd.or $\mathrm{g} /$ healtheqt/hospital-beds.htm [23.06.2020].

Prien, T.: Bestimmung des Therapieausmaßes - ethische und rechtliche Grundlagen, in: Intensivmedizin up2date 9 (2013) 120-140.

Seelmann-Eggebert, Kathrin: Die Flammenhölle von Ramstein (10.12.2018), in: www.spiegel.de/geschichte/ramstein-1988-die-flugschau-katastrophe-auf-der-us-a ir-base-a-1242058.html [28.05.2020].

Sefrin, P.: Sichtung - zentrales Element zur Bewältigung eines Großschadens und einer Katastrophe, in: Notarzt 28 (2012) 194-202.

Spiegel.de: ICE-Unglück von Eschede, in: www.spiegel.de/thema/ice_unglueck_vo n_eschede/ [28.05.2020]. 
Straub, Dominik: Italiens Trauma nach der Havarie der Costa Concordia (12.01.2017), in: www.tagesspiegel.de/gesellschaft/panorama/schiffsunglueck-vo r-fuenf-jahren-italiens-trauma-nach-der-havarie-der-costa-concordia/19246770.ht $\mathrm{ml}[28.05 .2020]$.

Steiermark.orf.at, Streit um Therapiekosten für schwer kranken Bub (29.01.2018), in: https://steiermark.orf.at/v2/news/stories/2892085/ [23.06.2020].

Taupitz, J.: Verteilung medizinischer Ressourcen in der Corona-Krise: Wer darf überleben?, in: MedR 38 (2020) 440-450. 
